\title{
Towards an Off-the-cloud loT data processing Architecture via a Smart Car Parking Example
}

\author{
Badraddin Alturki \\ Department of Computer Science \\ University of Leicester, UK \\ baba1@le.ac.uk
}

\author{
Stephan Reiff-Marganiec \\ Department of Computer Science \\ University of Leice ster, UK \\ srm13@le.ac.uk
}

\begin{abstract}
Nowadays, it is obvious that technology has revolutionised our lives by supporting us to do complicated jobs. The Internet of Things (IoT) is one of the emerging technologies. One of the most significant current research topics in the IoT is smart city. The smart city includes several applications such as smart home, smart industry and smart mobility. The smart car parking system is an aspect of smart mobility and an important application in smart city projects, because of the rapidly increasing number of cars in urban areas. However, most of the current proposals in smart car parking systems manage the data on the cloud side which is a problem since the system needs to send the raw data from sensor to cloud and receive instructions back: this is expensive in terms of energy and data transmission cost. To tackle this issue we present a proposal to save energy and to reduce the amount of data that is transmitted over the network to cloud by processing closer to source in this paper. The architecture is demonstrated through a case study.
\end{abstract}

\section{Categories and Subject Descriptors}

- Information systems $\rightarrow$ Data management systems

\section{Keywords}

Internet of Things (IoT); smart city; smart car parking; middleware.

\section{INTRODUCTION}

A significant number of works have been presented by many companies and researchers in the field of IoT. The Internet of things (IoT) extends the vision of the internet by connecting all disconnected objects to the web. The IoT has been defined by the cluster of European research projects on the internet of things "The IoT allows people and things to be connected Anytime, Anyplace, with Anything and Anyone, ideally using Any network and Any service [9]". The IoT is becoming feasible with the invention of low power and cheap devices that have enough capabilities to sense, actuate, collect, store and process the sensor data.

Permission to make digital or hard copies of all or part of this work for personal or classroom use is granted without fee provided that copies are not made or distributed for profit or commercial advantage and that copies bear this notice and the full citation on the first page. To copy otherwise, or republish, to post on servers or to redistribute to lists, requires prior specific permission and/or a fee.
Smart traffic management builds on the sensing capabilities of IoT and addresses the needs of increased traffic. It is expected that the number of cars in the world will increase significantly from 841 million cars in 2008 to just over 1.6 billion cars in 2035 [13].

However, there are a limited number of car parking areas in most cities and because of this, the roads will be more crowded and people might not be able to find car parking. Consequently, there are a number of people who are disappointed and sometimes park on slots that are not reserved for ordinary people [4]. Therefore, it is important to reduce the crowded traffic in metropolitan cities and serve better services to the user by utilising the available car parking in the most efficient way [12]. Moreover, most car parking system has the ability to monitor the entry and exit gates [7]. However, these car parking systems do not focus on a method to detect the exact car parking slot in an effective and easy way. Therefore, there is a need for smart car parking to solve and control these issues and give better facilities to users. It is widely accepted that there is a significant problem in smart car parking project which is how to find the exact available vacancies for parking, made more complex by the fact that departures and arrivals happen quickly and frequently. Current approaches, where they exist, collect data and send it to cloud processing centres which determine solutions and provide these back to the car parks.

Questions of how to reduce such data transmission cost and how to save energy as well as provide information more quickly are important and require urgent answers. Every system in the IoT field produces a great amount of data by using sensors and other data producers showing that there is a more general need to process these large amounts of data in efficient ways. There are several steps to process the data in IoT including collect, filter, fuse, pre-process, store and deliver the data [10]. The data filtering function filters the sensor data to prevent the large amount of data that are transmitted over the network. In addition, Data Fusion function aggregates and integrates sensor data to have richer information and thus produce more accurate and meaningful.

Researchers have shown an increased interest in data fusion and filtering in the field of IoT. Data processing can be done at source, in the network and in the cloud. The research to date has tended to focus on cloud processing rather than in-network level processing. It is clear that cloud computing is important for enabling the vision of IoT from several perspectives such as data storage and high data processing power [11], but this can be enhanced by IoT middleware which supports data management including data processing and real-time services to IoT applications [6] in other parts of the network: i.e. of-the-cloud!

It is important to know where data processing will be done most effectively. We consider that processing sensors data locally with 
middleware before sending to the cloud for further processing and storage is highly relevant for the internet of things and thus largely overlooked.

In this paper, the main contributions are: a) an approach to filter sensors data to discover the existence of vehicles on a parking slot in smart car parking by using wireless sensor network through localised processing and b) a middleware architecture which can manage the generated data from sensors to support the approach. The paper is driven by the car parking scenario, but the ideas are more general.

The rest of this paper is organised as follows. First of all, we give a number of challenges and highlight open questions. We then introduce the proposed approach and architecture based on the IoT. Section 4 provides a description of the case study and how this maps to the architecture. Then, we present some observations and the related works. Finally, we give the summary and highlight the future work.

\section{CHALlengeS}

There are a number of main challenges in smart city projects including data fusion and filtering [10]. Data fusion techniques allow to aggregate and integrate sensor data from several sources to produce knowledge. Data filtering is important to minimize the amount of data that are transmitted over the network [10].

However, where and how to conduct data fusion and filtering are difficult issues. In-network level data processing is important because the unused, unnecessary and private data is not going to be transmitted over the network. In other words, the continuously generated raw sensor data are not sent to the cloud, which is costly and expensive.

In this paper we focus on data filtering, thus trying to answer challenges around when it is right to retain data and when it can be safely discarded (the filtering challenge) considering the smart parking application.

\section{PROPOSED APPROACH}

In this section, we describe the structure of smart car parking system which includes the sensor node, smart lane object (sink node), cloud, smart floor object (data management middleware) and smart car parking facilities. This system has the following functionalities consisting of processing the sensors data locally, displaying the availability of car parking slots, able to guide the driver to find the location of the parking slot. In terms of communication wireless communication is used between sensors and smart objects based on technologies such as Bluetooth, ZigBee and WIFI.

As we discussed in Section 1, there are several steps of data management in IoT, from collection to analysis and results presentation. In this part, we are going to focus on the data filtering approach and explain the methods of the system. There are two types of smart objects: the first one is a smart lane object and the second one is a smart floor object. They have common capabilities such as communication facilities, data management, ad-hoc and device manager. However, they differ in storage, speed and processing level capability. Figure 1 shows the architecture and functionalities of the system and details are explained below.

\subsection{DATA FILTERING APPROACH}

Primary Filtering (PF) is a function that filters the sensor data according to the car parking slots availability. A good illustration of this is to check the status (free/full) of the sensors if the state is changed, then turn on the sensor reading otherwise turn it off.

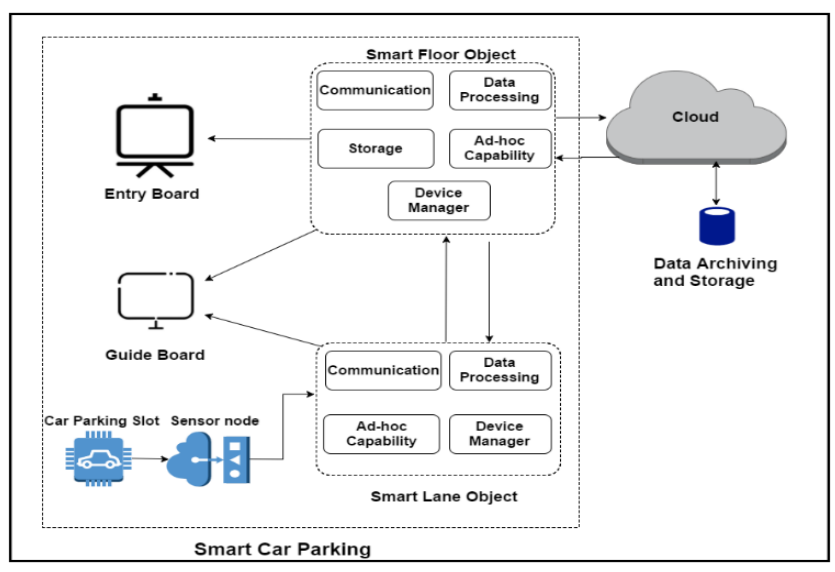

Figure 1. Functionalities of Smart Car Parking

Secondary Filtering (SF): is a function that filters the data depending on parameters (floor number, lane number, park number, time, date, status and plate number) and it has two types of filtering methods as follows:

- Availability Based Filter (ABF): It filters the data based on the status and floor number which are the most important aspects and calculates the number of free spaces because there is a need to show the number of the availability in the entry board.

- User Priority Based Filter (UPBF): it filters the sensor data based on the preferences and priorities that the users have identified when they are registered. According to the preferences that are set by the user this function calculates the relevant free parking spaces.

\section{Sensor node}

This node is the lowest level of the smart parking system and it is placed in every parking slots. It senses the parking slot to check the presence of car by the light sensor. Then, it sends the data to smart lane object by using wireless communication. Furthermore, there are LEDs work with a light sensor to indicate the status of the parking spaces. Additionally, there are LEDs the red one means the status is full and the green one means the status is free.

\section{Smart Lane Object}

It acts as sink node and it has low processing power and low storage but is good at real time processing. It is responsible for collecting sensors data using wireless communication and it does Primary Filtering (PF) on the collected data. This step is important to save energy and reduce the data transmission over the network. In addition, Device Manager is used for turning on and off the sensor reading according to PF function. In addition, this device manager is a standard interface supported by the company that built the platform. In addition, the sink node sends the data that contains available vacancy and status of car slots to the Smart Floor Object using wireless ad-hoc network. Additionally, it sends the information of available park number to guide board to guide the driver.

\section{Entry board}

The board has an electronic counter and an electronic sign that show the number of the available car park on every floor. 
Additionally, this board gets the information from Smart Floor Object to show the number of availability slots in each floor.

\section{Guide board}

It is an electronic board and it displays the empty slots in a screen that show the directions and the parking numbers. In addition, it gets the information from the Smart Lane Object and smart floor object to show the user the exact vacancy number.

\section{Smart Floor Object}

It is a data management middleware and it has the required storage to save data and the power to process data locally. Furthermore, this is the main method of the system which is the brain of the system. In addition, it collects the data from the smart lane object using ad-hoc wireless network to do SF function on the primarily filtered data and it stores the data locally for specific period of time. In addition, it sends the processed data which contains the number of free car parking slots to the main entry board and the entry of each floor for displaying the availability of car parking space. Furthermore, this method sends the data to the cloud using web protocols such as HTTP or COAP for storing and archiving the data. In addition, it can also send data to guide board on specific conditions such as registered users and failure of sink node.

\section{CASE STUDY}

Most shopping malls and airports in the world as well as most cities have multi-storey car parking. In addition, almost all of them have an automated system in terms of ticketing which helps to show the availability of parking spaces every time the car enters or exits the parking. However, it does not show exactly where are the available vacancies. Thus, a number of people may argue that the journey to find a park consumes gas and time. Therefore, it is crucial to have a car parking system that deals with these issues and monitors the availability of car parking to give better services to drivers. The smart car park will be equipped with smart lane object, smart floor object, entry board, guide board, sensors, traffic lights, LEDs, electronic signs, electronic counter and car plates reader as shown in Figure 2.

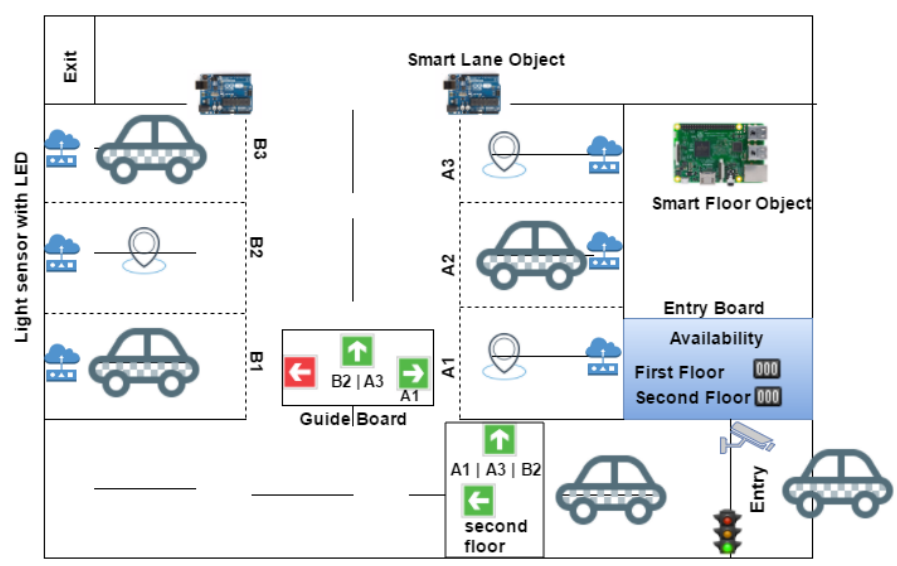

Figure 2. Case Study of Car Parking

In this section, we give a detailed description of the case study and how this maps to the architecture from section 3 .

\section{Plates Reader}

It is a camera that detects the plates number which is already done by many projects which is Automatic number plate recognition system. We use this reader just to check whether the car is registered with the system or not. The acquired data from plates reader are sent to smart floor object for processing.

\section{Smart lane object:}

This module collects the sensor data that is related to the specific lane and it does PF function on the raw sensor data. In addition, after applying primary filtering function on sensor data we identify the available and occupied car parking vacancy that are needed to send to guide board to show the park number to users. In addition, this information which contains several parameters (floor number, lane number, park number, time, date and status) are sent to smart floor object for further processing to extract more meaningful information.

Scenario: there are six car parking slots in the car park which contains $\mathrm{A}$ to $\mathrm{C}$ lanes and park number from 1 to 3 . First example, the car parking B1 was occupied from 10 am to $10 \mathrm{pm}$; in this period of time the system considers that the status is not changed and it turned off the sensor reading. Similarly, the park A1 is free from 10 am to $12 \mathrm{pm}$ which is considered as unchanged status and the status is already sent to the system when it was free. However, car park A2 changes frequently from free to full and conversely. In this case, the system turns on the sensor reading. If this smart object fails, then the smart floor object takes the responsibility of guiding the driver.

\section{Smart Floor Object:}

It gets primarily filtered sensor data from sink node with several parameters (floor number, lane number, park number, time, date and status) and the data from plates reader which is used to check whether the car is registered or not. Additionally, if the user is registered in the system, then the smart floor object takes the responsibility of guiding the driver by showing the available car park numbers in the guide board. Furthermore, a Floor, lane and park numbers parameters are used to find the location and exact place of the sensor. Additionally, time and date parameters are used to compare the new data with the old one. The last parameter is status which is important to show the available vacancies in the parking area for drivers in the main entry and in the entry of every floor. Moreover, it does Secondary Filtering (SF) function on the primarily processed data to identify the available spaces and calculate them to show the results in the entry board and store it locally.

Scenario: this scenario shows when user is registered with the system. The system allows the users to select their priority from 1 to 5 from very low to very high. Firstly, the system filters the data according to parameters (floor, lane and park number). Then, according to the user preferences and priorities that are identified, the floor number is set to 1 as a very high priority, lane is set to A as a moderate priority and the park number is set to 1 or 2 as low priority. According to the criteria, the system filters the floor 1 first because it has the highest priority. Then, it filters the lane A and at the end it filters the park number from 1 to 2 . This process is done based on the priorities and preferences, and it used to show the user the right place to park.

\section{RELATED WORK}

In this part, we show the literature review that includes the works that have been done on smart car parking system. [2] proposed a car parking system to find the available parking space depending on the visual sensor network. In addition, there are wireless cameras installed in the local area to get the image of the parking lots and send them to a centralised system for processing. They demonstrated promising outcomes by deploying the system on a 
real visual sensor network and the test presented how good their method in terms of performance and low requirements. However, this system generates a significant amount of data and this data will be transmitted over a network to a centralised system that will increase the transmission cost of data and consume more energy.

[5] proposed a cloud-based car parking method to reduce the consumption of time and gas while finding car parking lots. Additionally, they used simulation tools to show their system architecture and they have implemented their system in a real scenario. They are using wireless sensor network and RFID to control the car park that produces a large amount of data which are sent to the cloud for processing. Whereas, they did not concern about the huge generated data that are going to be sent to the cloud which is expensive.

[3] showed a prototype for displaying the available vacancies in the car park with the support of IoT. In addition, they used the online reservation system to book the parking slot in advance. However, they did not mention about guiding the user who is not booked in advance the car parking slot on the internet.

Finally, the work in [8] proposed car parking system in IoT in the context of airport car parking which allows the registered users to login to the system to check the free spaces in the parking. In addition, GPS is used to show the exactly available vacancy in the parking area to users using cloud server. However, all the processed data is transmitted to cloud for processing then the results sent to the users. This means a lot of data is transmitted over the network which is high cost of data transmission.

None of the existing approaches attempts to localize data processing by moving some decision making closer to the source of the data. We have proposed a data management approach to process sensor data locally to avoid the high volume of data to be transmitted over the network and we used WSN to monitor the car parking slots. Furthermore, a data filtering technique is used to reduce the energy consumption and cost of data transmission in our approach.

\section{DISCUSSION}

The smart car parking data management system is provided way to save energy and reduce the data transmission over the network. There are a number of advantages of this system. The first advantage is to help the users to save time by providing the right direction of the free car parking slot effectively. Another positive aspect is that the system is automated which does not require any user intervention. Additionally, using in-network level data processing will increases the privacy by isolating the system from global world because the data processing and storage is done locally rather than sending all the data to cloud for processing.

However, if we would try to extend our approach to a larger number of parking areas i.e. all parking areas in a neighbourhood or city, then a number of problems might appear which could be a consequence of the system. Trying to use this approach on larger scale we should keep track of the knowledge of the surrounding parking areas, something that we do not consider in our system. This would result in different trade-offs like, allowing more local hardware processing or more interaction with the cloud. The right balance would be crucial in terms of resource cost, energy consumption and data transmission.

After discussing the advantages and limitations of the system, this system is suitable for small to medium scale car parking. However, it can be extended to larger scale car parking system by scaling the system architecture to cope with large scale car parking.
We are implementing data filtering approach depending on java platform, then we are going to evaluate the processing time and energy consumption.

\section{CONCLUSION AND FUTURE WORK}

This paper has considered an approach to find the available vacancy in car parking in the field of IoT. The most obvious finding to emerge from this study is that we proposed a data management method that can be added to a middleware that has the capability to process the sensor data locally to save energy and reduce the data transmission over the network. We showed the proposed approach of the system and its relevant methods. In addition, we used a case study to show how this work maps to the architecture. On the basis of the promising findings presented in this paper, work on the remaining issues is continuing and will be presented in future papers. Future work will involve implementing the proposed approach and test the system to check the results. Then, extending this smart car parking system to larger scales (e.g. city) to test the scalability of the system. Finally, developing a mobile application for users to find the available parking slot and guide them through this application.

\section{References}

[1] M. Abu-Elkheir, M. Hayajneh, and N. A. Ali. Data management for the internet of things: Design primitives and solution. Sensors, 13(11):15582-15612, 2013.

[2] L. Baroffio, L. Bondi, M. Cesana, A. E. Redondi, and M. Tagliasacchi. A visual sensor network for parking lot occupancy detection in smart cities. In Internet of Things (WF-IoT), 2015 IEEE 2nd World Forum on, pages 745-750. IEEE, 2015.

[3] B. M. K. Gandhi and M. K. Rao. A prototype for iot based car parking management system for smart cities. Indian Journal of Science and Technology, 9(17), 2016.

[4] L. Mainetti, L. Patrono, M. L. Stefanizzi, and R. Vergallo. A smart parking system based on iot protocols and emerging enabling technologies. In Internet of Things (WF-IoT), 2015 IEEE 2nd World Forum on, pages 764-769. IEEE, 2015.

[5] T. N. Pham, M.-F. Tsai, D. B. Nguyen, C.-R. Dow, and D.-J. Deng. A cloud-based smart-parking system based on internet-of-things technologies. IEEE Access, 3:1581-1591, 2015.

[6] M. A. Razzaque, M. Milojevic-Jevric, A. Palade, and S. Clarke. Middleware for internet of things: a survey. IEEE Internet of Things Journal, 3(1):70-95, 2016.

[7] S. Srikanth, P. Pramod, K. Dileep, S. Tapas, M. U. Patil, et al. Design and implementation of a prototype smart parking (spark) system using wireless sensor networks. In Advanced Information Networking and Applications Workshops, 2009. WAINA'09. International Conference on, pages 401-406. IEEE, 2009.

[8] M. Suresh, P. S. Kumar, and T. Sundararajan. Iot based airport parking system. In Innovations in Information, Embedded and Communication Systems (ICIIECS), 2015 International Conference on, pages 1-5. IEEE, 2015.

[9] O. Vermesan, P. Friess, P. Guillemin, S. Gusmeroli, H. Sundmaeker, A. Bassi, I. S. Jubert, M. Mazura, M. Harrison, M. Eisenhauer, et al. Internet of things strategic research roadmap. Internet of Things: Global Technological and Societal Trends, 1:9-52, 2011. 
[10] M. Wang, C. Perera, P. P. Jayaraman, M. Zhang, P. Strazdins, and R. Ranjan. City data fusion: Sensor data fusion in the internet of things. arXiv preprint arXiv:1506.09118, 2015.

[11] A. Zaslavsky, C. Perera, and D. Georgakopoulos. Sensing as a service and big data. arXiv preprint arXiv:1301.0159, 2013.

[12] Y. Zheng, S. Rajasegarar, and C. Leckie. Parking availability prediction for sensor-enabled car parks in smart cities. In Intelligent Sensors, Sensor Networks and Information Processing (ISSNIP), 2015 IEEE Tenth International Conference on, pages 1-6. IEEE, 2015.

[13] Organization of the Petroleum Exporting Countries (OPEC), 2011. World Oil Outlook 2012. At: http://www.opec.org/opec web/static files project/media/d ownloads/publications/WOO2012.pdf 